Revista de

Contabilidade e

Organizações

www.rco.usp.br
DOI: http://dx.doi.org/10.11606/rco.v11i29.122140
Journal of

Accounting and

Organizations

\title{
O efeito do treinamento na percepção do desempenho individual: uma análise baseada nos treinamentos ofertados pelo Instituto Federal do Espírito Santo (IFES)
}

\author{
Katia Cristina Cabral Monteiro Galvao ; Danilo Soares Monte-mor ${ }^{\mathrm{a}}$; Neyla Tardin ${ }^{\mathrm{a}}$
}

${ }^{a}$ Fucape Business School

\section{Informações do Artigo}

Histórico do Artigo

Recebido: 20 de outubro de 2016

Aceito: 13 de abril de 2017

Palavras-chave:

Treinamento.

Desempenho.

Setor Público.

\begin{abstract}
Resumo
Este artigo investiga se os treinamentos ofertados aos servidores públicos melhoram o seu desempenho no cumprimento de suas atribuições. Para tanto, optou-se por uma Instituição Pública de Ensino, cuja ampliação de suas unidades entre 2008 e 2010 resultou no aumento do quadro de pessoal e na criação da capacitação obrigatória para os novos servidores por meio da Ambientação Institucional. A partir de um modelo de diferenças em diferenças, estimou-se o efeito do treinamento no desempenho individual dos servidores que participaram do treinamento, controlando-se por alterações no desempenho de servidores não treinados e por outras características heterogêneas. Os resultados obtidos sugerem que o treinamento não analisado teve impacto sobre o desempenho individual de servidores públicos, corroborando a hipótese de que treinamentos não necessariamente aumentam o desempenho.
\end{abstract}

Copyright (C) 2017 FEA-RP/USP. Todos os direitos reservados

\section{INTRODUÇÃO}

Reformas promovidas na Administração Pública de diversos países resultaram na necessidade de modernização dos processos do setor público e no fomento ao desenvolvimento contínuo de pessoal (BresserPereira, 2010). Diante da rejeição ao papel intervencionista do Estado e da globalização de mercados, foi preciso buscar referências já experimentadas no setor privado, adequando os modelos utilizados para a realidade do setor público, com o objetivo de uma gestão mais eficaz, por meio de redução de custos e foco na obtenção de melhores resultados (Peci, Pieranti \& Rodrigues, 2008; Klering, Porsse \& Guadagnin, 2010; OCDE, 2010a).

Nessa perspectiva, ao redor do mundo, o sistema de gestão New Public Management (NPM) vem orientando tais reformas e promovendo avanços graduais via integração entre as dimensões políticas e administrativas (Motta, 2013; Verbeeten \& Speklé, 2015). De forma similar, o governo brasileiro promoveu em 2005 o lançamento do Programa Nacional de Gestão Pública (Gespública) com a finalidade de alcançar resultados mais eficazes. Tal Programa foi acompanhado em 2006 pela instituição da Política Nacional de Desenvolvimento de Pessoal (PNDP), que introduziu a gestão por competências como instrumento gerencial do processo de desenvolvimento contínuo e permanente de pessoal (Fonseca et al., 2013; Filardi et al., 2016 ).

A despeito dessas iniciativas, no Brasil, existem evidências de carência de competências para o desempenho de funções, resultado da ausência de um sistema moderno de gestão de pessoas (Jãrvalt \& RandmaLiiv, 2010; Junior, Zouain \& Almeida, 2014). Tais evidências sugerem uma lacuna em relação à efetividade do modelo de gestão estratégica de recursos humanos, principalmente no que se refere à eficácia de treinamento de pessoal (Salas et al., 2012; De Grip \& Sauermann, 2012; De Grip \& Sauermann, 2013), tal como expresso nas recomendações do Tribunal de Contas da União, sobre os controles nos gastos públicos com capacitação de pessoal (Acórdão 866/2011-TCU - Plenário).

A partir dessas primeiras considerações, este artigo tem o objetivo de verificar se a capacitação de pessoal tem contribuído para suprir a carência de competências para o desempenho de funções em instituições públicas.

Autor Correspondente: Tel (27) 4009-4444

E-mail: katiacgalvao@ifes.edu.br (K. C. C. M. Galvao); danilo@fucape.br (D. S. Monte-mor); neylatardin@yahoo.com.br (N. Tardin)

FUCAPE - Av. Fernando Ferrari, 1358 - Boa Vista, Vitória - ES, 29075-505, Brasil. 
Especificamente, pretende-se investigar se os treinamentos ofertados aos servidores públicos promovem melhoria na precepção sobre o desempenho individual no cumprimento de suas atribuições.

A literatura econômica por vezes é conflitante acerca dos efeitos de treinamentos sobre o desempenho. Alguns estudos mostram que investimentos em treinamento podem aumentar o desempenho individual e organizacional e trazer benefícios tanto para o trabalhador quanto para o empregador, dado o desenvolvimento de competências e a promoção de atualização profissional gerada pelos treinamentos (De Grip \& Sauermann, 2012; Verbeeten \& Speklé, 2015). Nesse sentido, treinamentos no local de trabalho podem resultar em efeitos sobre a produtividade do trabalhador e servirão de sustentação para o crescimento das organizações (Ariga et al., 2013).

Por outro lado, outros autores afirmam que não se observa necessariamente aumento de desempenho a partir de treinamentos (De Grip \& Sauermann, 2013; Lopes \& Teixeira, 2013). Para que tais investimentos mostrem resultados tangíveis para as organizações, os programas de treinamento devem ser adequadamente projetados e sua implementação posteriormente avaliada (Patel, 2010; Salas et al., 2012). Nesse caso, aponta-se a necessidade de se estipular critérios de desempenho que estejam alinhados aos objetivos do treinamento, a fim de aumentar a eficácia dos treinamentos realizados (De Grip \& Sauermann, 2013).

Para que fosse possível investigar os efeitos de treinamentos sobre o desempenho individual de servidores públicos, adotamos como corpus da pesquisa uma Instituição Pública de Ensino que passou por um processo de ampliação de suas unidades educacionais entre 2008 e 2010, que resultou no aumento do quadro de pessoal e na criação da capacitação obrigatória para todos os novos servidores - Ambientação Institucional (Ambientação).

Os dados relativos à participação em treinamentos da Ambientação e das avaliações de desempenho foram obtidos nos respectivos sistemas da Instituição. Foram criados dois grupos, um para identificar servidores que participaram de treinamentos (Tratamento) e outro com servidores que não participaram (Controle). A partir dos dados dos grupos de tratamento e de controle, estimou-se o efeito do treinamento no desempenho individual. Os testes foram controlados por outros efeitos heterogêneos que poderiam influenciar o desempenho individual, como autoavaliação, avaliação do usuário, além de tempo de serviço na Instituição, idade e gênero. Corroborando com as evidências presentes em De Grip e Sauermann (2013) e Lopes e Teixeira (2013), os resultados obtidos sugerem que os treinamentos analisados não tiveram impacto sobre o desempenho dos servidores treinados.

Dessa maneira, este artigo soma-se a outros estudos sobre treinamento nas organizações que destacam a necessidade de se estipular critérios de desempenho que estejam alinhados aos objetivos do treinamento, a fim de aumentar a eficácia dos treinamentos realizados (Patel, 2010; Salas et al., 2012; De Grip \& Sauermann, 2013; Lopes \& Teixeira, 2013). Especificamente, esta pesquisa se diferencia desses trabalhos ao conduzir a investigação considerando treinamentos ofertados a trabalhadores do setor público, no qual o mecanismo de incentivos e as características do ambiente organizacional se diferem dos utilizados no setor privado (Agrawal, 2013).

Dentre as pesquisas conduzidas no setor público, Mendez, Sepúlveda, e Valdes (2015) identificaram que treinamentos tiveram associação positiva com salários dos trabalhadores tanto do setor público quanto do privado em países desenvolvidos. Brandão, Borges-Andrade e Guimarães (2012), em um estudo em um banco de economia mista, encontraram resultados que demonstraram que horas de treinamento podem influenciar positivamente o desempenho organizacional. Da mesma forma, Amorim e De Barros Silva (2013) concluíram que houve efeito positivo dos treinamentos na performance sob a perspectiva dos servidores de uma Universidade Federal.

Este trabalho se diferencia desses estudos ao encontrar evidências de que não necessariamente treinamentos aumentam o desempenho de servidores do setor público. Nesse caso, tal resultado sugere um alerta para a necessidade da estipulação de diretrizes, mecanismos de controle, estímulo e incentivos para que servidores públicos não só realizem treinamentos, mas apliquem e demonstrem seus efeitos como contrapartida aos recursos investidos que, por serem limitados, exigem políticas e práticas de gestão de pessoas que sejam efetivas e eficazes.

\section{REFERENCIAL TEÓRICO}

\subsection{Treinamento e produtividade nas organizações}

A Gestão de Recursos Humanos (GRH), ao longo dos anos, tem passado por mudanças em suas funções e, gradativamente, vem alcançando papel estratégico nas organizações (Coda \& Coda, 2014). Acrescente-se, a 
esse cenário, a constatação de que, depois da educação formal, o treinamento promovido em local de trabalho é uma das formas de melhorar o desempenho do trabalhador; por consequência, contribuir para taxas de crescimento econômico (Aguinis \& Kraiger, 2009; Ariga et al., 2013; Fonseca et al., 2013).

Nesse contexto, organizações que investem na formação de trabalhadores desejam obter mudanças sustentáveis de comportamento e aprendizado de competências que garantam vantagem competitiva (Salas et al., 2012; Ariga et al., 2013). Também no serviço público, esses objetivos são desejáveis, especialmente em razão da limitação de recursos, o que exige eficácia das políticas de GRH (Salas et al., 2012).

Em países desenvolvidos, como Estados Unidos e Reino Unido, pesquisas indicam que treinamentos afetam produtividade e desempenho, tanto no setor privado quanto público (De Grip \& Sauermann, 2012; Colombo \& Stanca, 2014; Guerrazzi, 2016). Isso ocorre porque nesses países a legislação trabalhista permite a existência de remuneração variável, inclusive para trabalhadores do setor público (Mendéz, Sepúlveda, \& Valdés, 2015). Nesse ponto, destacamos que a remuneração por desempenho funciona como um mecanismo de incentivo ao aprendizado e ao aumento de desempenho.

No serviço público japonês, por exemplo, de acordo com Koga (2014), a remuneração dos servidores públicos é equiparada à dos empregados do setor privado, mediante correlação das atribuições, tempo de serviço e escolaridade dos trabalhadores. Assim, são aplicados os mesmos percentuais de aumento ou redução salarial sobre as remunerações de trabalhadores, dos setores público e privado, conforme o desempenho do mercado e, por consequência, arrecadação de tributos, que em última instância funcionam como fonte dos recursos públicos (Koga, 2014).

Em países, como o Brasil, um dos principais problemas do serviço público são as características de instituições e de planos de carreiras que não permitem a implementação de sistemas de remuneração variável (Agrawal, 2013). Nesse caso, muito embora em algumas funções o setor público pague em média valores superiores ao do setor privado e atraia profissionais com elevado potencial, a ausência de remuneração variável pode se configurar como falta de incentivo e desmotivação aos trabalhadores, comprometendo a eficiência e desempenho individual e a exploração de toda a potencialidade desses servidores (Nakayama \& Salott, 2014). Nesse contexto, as organizações públicas tendem a ficar sob a influência do risco moral, uma vez que o desempenho dos trabalhadores, sem mecanismos de incentivos adequados, fica condicionado às motivações pessoais a despeito dos objetivos das próprias organizações (Klann et al., 2015).

Balsan et al. (2016), por exemplo, afirmam que o treinamento isoladamente não é capaz de implementar e sustentar impacto positivo no desempenho, mas que a associação a estratégias pós-treinamentos pode resultar em alto impacto positivo no trabalho. Além disso, outros estudos sugerem que gestores devem adotar atitudes que incentivem a participação dos trabalhadores, criando ambientes favoráveis à aplicação do aprendizado nas organizações (Chiaburi, Van Dam, \& Hutchins, 2010; Salas et al., 2012; Mcnamara et al., 2012; Kumpikaitė, 2015). Em outros termos, mecanismos de incentivos adequados e um ambiente favorável à aplicação do aprendizado proporcionará a transferência de aprendizado, que contribui positivamente para a motivação e para o aprendizado dos indivíduos submetidos a treinamentos (Grossman \& Salas, 2011). Com isso, haverá indivíduos motivados a participarem de treinamentos e ao autodesenvolvimento no trabalho (Martin, 2010; Salas et al., 2012).

Aliado ao problema de risco moral causado pela ausência ou ineficiência da política de incentivos, a literatura aponta pela necessidade do desenvolvimento de políticas de planejamento e gestão do ambiente organizacional para a eficácia dos treinamentos. Isso porquê o sucesso do treinamento não depende apenas dos mecanismos de incentivo, mas da qualidade do seu programa e das variáveis de contexto. A correlação entre as variáveis de qualidade que dão suporte à aprendizagem e à performance no trabalho, tal como analisado por Balarini, Zerbini e Martins (2014), corroboram a necessidade de conciliação de motivação, qualidade e incentivos individuais para o sucesso de treinamentos.

Nesse caso, verifica-se que o treinamento não é um evento isolado e único, mas um processo sistêmico, que, se bem projetado, funcionará como estímulo às várias métricas de bem-estar corporativo (Salas et al., 2012). Por isso, a formulação, a implementação e a avaliação de treinamentos devem ser conduzidas a partir de decisões adotadas visando a eficácia de treinamentos, tanto sob o ponto de vista dos mecanismos de incentivo quanto sob o ponto de vista da qualidade do treinamento e do ambiente organizacional.

Observadas tais situações, seja pela falta de incentivos à melhoria do desempenho ou pela ausência de qualidade no ambiente e no próprio treinamento, é possível que o treinamento não tenha efeito sobre a melhoria 
do desempenho dos servidores públicos brasileiros. A partir disso, a presente pesquisa testa a seguinte hipótese: públicos.

H1: Treinamento não necessariamente tem efeito positivo sobre o desempenho individual de servidores

\section{METODOLOGIA}

\section{1 Seleção da amostra e descrição do modelo}

Para que fosse possível investigar os efeitos de treinamentos sobre o desempenho individual de servidores públicos, utilizou-se um treinamento realizado por uma Instituição Pública de Ensino que passou por um processo de ampliação de suas unidades educacionais entre 2008 e 2010. Tal ampliação resultou no aumento do quadro de servidores e na implantação da capacitação obrigatória para todos os novos servidores - Ambientação Institucional (Ambientação).

A população da pesquisa envolveu servidores integrantes da carreira dos Cargos Técnico Administrativos em Educação - TAE, em exercício nas várias unidades educacionais do Instituto Federal do Espírito Santo (IFES), submetidos à avaliação de desempenho, conforme programa contido no Plano de Carreira. Os dados foram obtidos junto aos sistemas de avaliação de desempenho e do projeto Ambientação, e estruturados em painel. Os dados dos treinamentos utilizados referem-se aos Cursos de Desenvolvimento de Servidores (CDS). O projeto foi elaborado segundo as Diretrizes do Decreto n ${ }^{\circ}$ 5.707/06, que instituiu a Política Nacional de Desenvolvimento de Pessoal sob a perspectiva do modelo de desenvolvimento estratégico de pessoas (Brasil, 2006; Carvalho, 2009).

O projeto, executado no período de 2010 a 2012, teve seus objetivos alinhados à estratégia da Instituição, no qual profissionais da própria organização atuaram como instrutores selecionados a partir de saberes e competências comprovados por currículo na Plataforma Lattes do CNPq (IFES, 2016). O IFES constitui-se um ambiente favorável à implementação do projeto, dado que, por se tratar de instituição de ensino, conta com um quadro diversificado de especialistas, o que permitiu trabalhar com instrutores ligados à variedade de áreas de conhecimento abordadas nos treinamentos. Nesse período, foram ofertadas 180 turmas, 4.554 vagas e 2.430 horas de treinamento, o que representou aproximadamente $70 \%$ das oportunidades de capacitação promovidas no período pela Instituição. Todas as informações relativas ao projeto foram disponibilizadas no sítio da Instituição (IFES, 2016).

A amostra foi composta pelo total de 4.818 observações de avaliações, compreendendo 1.493 observações das avaliações dos indivíduos que foram treinados (grupo de tratamento) e 3.325 observações de indivíduos não treinados (grupo de não-tratamento). Dentre os indivíduos não treinados, 1931 observações se referiam à avaliações de servidores que ingressaram no quadro antes de 2008. Dado que o tempo de serviço pode influenciar o desempenho dos servidores, análises foram conduzidas comparando-se os indivíduos treinados com: i) todos os demais servidores não treinados (grupo de não-tratamento); ii) todos os servidores não treinados e que entraram no mesmo período que os servidores que receberam o treinamento (grupo de controle). Além disso, controlou-se pelo tempo de serviço de cada servidor.

A partir dos grupos de tratamento e de controle, o modelo a seguir foi utilizado para se verificar o efeito do treinamento no desempenho:

$$
\text { PARES }_{i t}=+\alpha_{\mathrm{i}}+\mu_{\mathrm{t}}+\text { Treinamento }_{\mathrm{i}}+\text { Treinamento }_{\mathrm{i}} \cdot \text { Tempo }_{t}+\text { Controles }_{\mathrm{it}}+\varepsilon_{i t}
$$

A variável Tempo assume valores iguais a 1 para avaliações de desempenho feitas após o treinamento, e 0 caso contrário. A variável Treinamento é uma dummy de grupo e assume valores iguais a 1 para indivíduos que receberam o treinamento (grupo de tratamento) e 0 para indivíduos que não foram treinados.

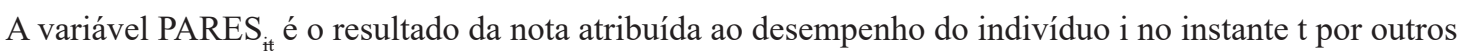
servidores do mesmo setor. Especificamente, a avaliação feita pelos pares é uma variável contínua e contempla 
avaliações feitas pelos demais servidores do mesmo setor. Tal avaliação é obtida por meio da aplicação de um questionário de avaliação aos servidores pares que contém 10 afirmações acerca de quesitos operacionais, organizacionais e comportamentais. A cada quesito é atribuída uma pontuação de 5, 10, 15 ou 20, correspondentes aos indicadores nunca, às vezes, quase sempre e sempre. Nesse caso, a pontuação máxima da avaliação feita por cada par é de, no máximo, 200 pontos. A média das avaliações feitas por cada par acerca de um dado servidor i é tomada como a avaliação de desempenho feita pelos pares para esse servidor.

Além disso, foram controladas por heterogeneidades associadas à autoavaliação do servidor (AutoAvaliação), avaliação do usuário do serviço (Avaliação-Usuário), e idade (Idade), gênero (Gênero) e tempo de serviço do servidor (Tempo Serviço). Foram incluídas dummies de tempo $\left(\mu_{t}\right)$ e de local de trabalho (campus) de cada servidor $\left(\alpha_{i}\right)$. Mais detalhes sobre treinamento e avaliação de desempenho na Instituição pesquisada e os controles utilizados estão expostos nas subseções seguintes.

\subsection{O treinamento no IFES}

O treinamento utilizado nesta pesquisa foi o Aperfeiçoamento I - Ambientação Institucional (Ambientação), instituída como capacitação obrigatória para todos os novos servidores a partir da edição da Resolução $n^{\circ} 14 / 2008$, do Conselho Diretor da Instituição. A Ambientação é composta por 8 treinamentos obrigatórios, que deveriam ser feitos em até 2 anos e 8 meses a partir do ingresso no Órgão (IFES, 2016).

Embora obrigatória, a participação na Ambientação estava condicionada à iniciativa dos próprios servidores que, além de realizarem o cadastramento no aperfeiçoamento, também fariam a solicitação de inscrição em cada um dos 8 treinamentos (Quadro 1). O projeto não previa cadastramento e inscrição automáticos; além disso, não era possível prever a disponibilidade do servidor para ausentar-se do seu local de trabalho, a fim de participar dos treinamentos nas datas ofertadas (IFES, 2016).

Para minimizar essa limitação da Ambientação, segundo os responsáveis pelo projeto, os treinamentos foram ofertados nas unidades educacionais que compõem o IFES (Quadro 2) na intenção de aumentar a participação dos servidores, mitigar os deslocamentos de servidores para outras unidades, e ainda assim, permitindo a integração de pessoal inter-unidades, uma vez que a participação nas turmas não estava associada ao local de trabalho do participante, observado o limite de vagas por turma. Além disso, um mesmo treinamento era ofertado tantas vezes quanto necessárias para buscar atender à demanda de interessados cadastrados no treinamento.

Quadro 1. Aperfeiçoamento I - Ambientação Institucional

\begin{tabular}{ll}
\hline Aperfeiçoamento & \multicolumn{1}{c}{ Treinamentos obrigatórios } \\
\hline & Documentos Institucionais (8horas) \\
& Fundamentos da Ética (8horas) \\
& História das instituições federais de Educação Profissional e \\
& Tecnológica (EPT) e história da EPT no Brasil (12 horas) \\
& Legislação educacional geral e específica em EPT e políticas \\
públicas em EPT e proposta pedagógica institucional & \\
& Legislação Específica (Lei 8.112/90) - módulo I (16horas) \\
& Legislação Específica (Lei 8.112/90) - módulo II (8horas) \\
& Modelagem de Processos (16horas) \\
& Racionalização do Trabalho (16horas) \\
\hline Ambientionacional &
\end{tabular}

Fonte: http://pse.ifes.edu.br/cds/site/ Acesso em 27.jul.2016 
Quadro 2. Regiões para oferta dos treinamentos

\begin{tabular}{cl}
\hline Regiões & \multicolumn{1}{c}{ Unidades Educacionais } \\
\hline \multirow{3}{*}{ Central } & Microrregião Metropolitana: Reitoria e os Campi Cariacica, Guarapari, Serra, Vila \\
& Velha e Vitória \\
& Metrópole Expandida Sul:Campus Piúma \\
& Sudoeste Serrana:Campus Venda Nova do Imigrante \\
& Polo Linhares:Campi Aracruz e Linhares \\
Norte & Litoral Norte:Campus São Mateus \\
& Polo Colatina:Campi Colatina e Itapina \\
& Central Serrana:Campus Santa Teresa \\
Noroeste & Noroeste 2: Campus Nova Venécia \\
& Polo Cachoeiro:Campus Cachoeiro de Itapemirim \\
& Caparaó:Campi Alegre e Ibatiba \\
\hline
\end{tabular}

Fonte: http://pse.ifes.edu.br/cds/site/ Acesso em 27.jul.2016

\subsection{Avaliação de desempenho no IFES}

Instituído pela Lei no 11.091 (Brasil, 2005) e regulamentado pelo Decreto no 5.825 (Brasil, 2006), o Programa de Avaliação de Desempenho compõe o Plano de Carreira dos Cargos Técnico-administrativos em Educação - PCCTAE, aprovado pela Resolução do Conselho Diretor no 11/ 2007 (IFES, 2016). O Programa foi proposto como instrumento de gestão de pessoas, com vistas à melhoria da qualidade do trabalho, por meio do qual seria possível identificar a integração dos servidores com a Instituição, além de identificar problemas de supervisão (PCCTAE, 2007).

A avaliação de desempenho foi estruturada para contemplar três segmentos: o próprio servidor (Autoavaliação), a equipe de trabalho e a chefia (Hetero-avaliação), e o usuário (Avaliação-Usuário). Para cada segmento, são utilizados formulários para registrar a percepção do desempenho do servidor avaliado.

A auto-avaliação (Auto-Avaliação) se refere a notas atribuídas pelo próprio servidor sobre seu próprio desempenho. A hetero-avaliação (PARES), atribuídas em consenso (média) pela equipe de trabalho e chefia sobre o desempenho do avaliado. Participam dessa perspectiva além da chefia do avaliado, no mínimo, outros dois servidores que tenham exercício no mesmo setor/gerência ou diretoria que o servidor avaliado. Já a avaliação do usuário (Avaliação- Usuário) refere-se à média das notas atribuídas por usuários internos ao serviço prestado pela unidade organizacional onde servidor avaliado desempenha suas funções.

A avaliação de desempenho ocorre a cada 9(nove) meses, a partir da data de admissão do servidor, quanso onde busca aferir o desempenho do avaliado, atribuindo as pontuações 5, 10, 15 e 20, que correspondem aos indicadores nunca, às vezes, quase sempre e sempre. Cada formulário contém dez afirmações acerca de quesitos operacionais, organizacionais e comportamentais. Nesse caso, a escala utilizada permite alcançar a pontuação máxima de até 200 pontos em cada seguimento. Exige-se um mínimo de 120 pontos para que o servidor seja considerado apto no processo de avaliação, pré-requisito para a concessão de progressão por mérito.

A despeito da tentativa de aproximar-se do modelo de avaliação de 360 graus, na avaliação de desempenho no IFES, não é empregado mecanismo objetivo de desempenho produtivo do indivíduo (Bégin \& Véniard, 2013; Poister, Thomas, \& Berryman, 2013). Pelo que é possível constatar do programa de avaliação de desempenho, os quesitos são avaliados a partir de percepções subjetivas de cada um dos segmentos.

\section{RESULTADOS}

\subsection{Estatística descritiva e testes de diferença de médias}

A partir da coleta de dados foram selecionados indivíduos que participaram e que não participaram 
de treinamentos para comparação de seus desempenhos. Os indivíduos foram classificados quanto a quem fez treinamento e quem não o fez. Para isso, foi criada uma variável dummy Treinamento igual a 1para indivíduos que receberam treinamento, e 0 caso contrário. A estatística descritiva das variáveis observadas neste estudo consta no painel A da Tabela 1 a seguir. No painel B, encontram-se os resultados dos testes de diferenças de médias para as variáveis utilizadas entre os dois grupos de indivíduos.

No painel A, constata-se que entre os indivíduos que não fizeram treinamento estão os com idade mais avançada (41 anos em média) e os que têm mais tempo de serviço (11 anos em média). Entre os que fizeram treinamento, estão os mais jovens (36 anos em média) e que têm menos tempo de serviço ( 3 anos e meio em média). O fato de os servidores mais jovens e com menos tempo de serviço serem os que mais participaram de treinamentos pode ser consequência de a Ambientação ter sido instituída como obrigatória para os novos servidores (IFES, 2016).

\section{Tabela 1. Estatística descritiva e teste de diferença de médias}

\section{Painel A: Estatística descritiva}

Este Painel apresenta a caracterização dos indivíduos quanto a quem não fez o treinamento (grupo de tratamento) e quem não fez o treinamento (grupo de não-tratamento) com relação ao desempenho e demais variáveis utilizadas no estudo durante todo o período analisado. Definições das variáveis: PARES $\mathrm{itt}_{\mathrm{it}}$ resultado da nota atribuída ao desempenho do indivíduo $\mathrm{i}$ no instante $\mathrm{t}$ por outros servidores do mesmo setor; Treinamento $=1$ para indivíduos que receberam o treinamento, 0 caso contrário; Auto-Avaliação = percepção do próprio servidor sobre seu desempenho; Avaliação-Usuário =percepção média sobre o desempenho por parte dos usuários internos do serviço; Idade = idade do servidor; Tempo Serviço = tempo de serviço do servidor na organização; Gênero = 1 se Masculino e 0 se Feminino.

\begin{tabular}{lccccccccccc}
\hline & \multicolumn{3}{c}{ Não fez treinamento } & \multicolumn{4}{c}{ Fez treinamento } \\
\multicolumn{1}{c}{ Variável } & Obs & Média & $\begin{array}{c}\text { Desvio } \\
\text { padrão }\end{array}$ & Min & Max & Obs & Média & $\begin{array}{c}\text { Desvio } \\
\text { padrão }\end{array}$ & Min & Max \\
\hline Pares & 3325 & 192.99 & 15.85 & 48 & 200 & 1493 & 193.65 & 12.04 & 70 & 200 \\
Auto-Avaliação & 3325 & 191.08 & 22.30 & 30 & 200 & 1493 & 191.82 & 16.42 & 33 & 200 \\
AvaliaçãoUsuário & 3325 & 184.59 & 24.81 & 18 & 200 & 1493 & 183.64 & 21.66 & 40 & 200 \\
Idade & 3325 & 41.83 & 10.03 & 20 & 72 & 1493 & 36.28 & 7.56 & 22 & 64 \\
Tempo Serviço & 2752 & 10.78 & 8.81 & 0 & 31 & 1134 & 3.52 & 3.92 & 0 & 29 \\
Gênero & 3325 & 0.5413 & 0.4983 & 0 & 1 & 1493 & 0.5077 & 0.50 & 0 & 1
\end{tabular}

Painel B: Teste de diferença de médias das avaliações e demais variáveis

Neste Painel são apresentados os resultados do teste de diferença de médias comparando-se os servidores que fizeram o treinamento (grupo de tratamento) e os servidores que não fizeram o treinamento (grupo de nãotratamento) para todo o período analisado.

\begin{tabular}{|c|c|c|c|c|c|c|}
\hline \multirow[b]{2}{*}{ Variável } & \multicolumn{2}{|c|}{ Não fez treinamento } & \multicolumn{2}{|c|}{ Fez treinamento } & \multirow[b]{2}{*}{ Diferença } & \multirow[b]{2}{*}{$\mathbf{P}>\mathbf{t}$} \\
\hline & Média & Desvio Padrão & Média & $\begin{array}{c}\text { Desvio } \\
\text { Padrão }\end{array}$ & & \\
\hline Pares & 192.99 & 15.85 & 193.65 & 12.04 & $-0,6619$ & 0.150 \\
\hline Auto-Avaliação & 191.08 & 22.30 & 191.82 & 16.42 & $-0,7453$ & 0.247 \\
\hline AvaliaçãoUsuário & 184.59 & 24.81 & 183.64 & 21.66 & 0.9501 & 0.950 \\
\hline Idade & 41.83 & 10.03 & 36.28 & 7.56 & $5.5465 * * *$ & 0.000 \\
\hline $\begin{array}{l}\text { Tempo Serviço } \\
\text { Gênero }\end{array}$ & 10.78 & 8.81 & 3.52 & 3.92 & $7.2611 * * *$ & 0.000 \\
\hline
\end{tabular}

Fonte: elaborado pelos autores

*** representam coeficientes significativos a $1 \%$

O painel B mostra os resultados dos testes de diferenças de médias das avaliações dos dois grupos de indivíduos para todo o período analisado. Verifica-se que, embora existam diferenças entre os indivíduos que fizeram e não fizeram treinamento em relação à idade e tempo de serviço, os dois grupos não apresentaram diferenças significativas com relação às médias de desempenho quando analisado todo o período da amostra. Em particular, tal resultado está alinhado com o objetivo da Ambientação que visava promover treinamento introdutório aos novos servidores, para que esses pudessem ter melhor desempenho em suas funções. 
Além disso, é possível que os servidores com mais idade e mais tempo de serviço, apesar de poder fazer os treinamentos de forma optativa, talvez não tenham motivação para se capacitar, em razão da proximidade com a aposentadoria (Pantoja et al., 2012). Em um estudo cujo objetivo foi identificar o efeito de horas de treinamento com aspectos do desempenho organizacional (produtividade, taxa de inovação e taxa de rotatividade), foi obtido resultado de menor participação em treinamentos entre trabalhadores mais velhos, cuja natureza exata da relação, para os pesquisadores, estava associada a variáveis como contexto cultural em que a organização estava inserida (Mcnamara et al., 2012).

Tais resultados sugerem que, tal como em Salas et al. (2012), fatores como envelhecimento dos trabalhadores e presença de nova geração no ambiente de trabalho com motivações e expectativas distintas quanto à aprendizagem requerem das organizações uma abordagem diferenciada quanto aos planos de treinamento propostos (Salas et al., 2012).

Na variável Avaliação-Usuário, observa-se maior variação entre as notas mínimas (18 pontos em média) atribuídas ao grupo de não treinados, e máximas (200 pontos em média) atribuídas ao grupo que fez treinamento. Essa variável captura a percepção de desempenho organizacional, dado que a nota é atribuída ao setor (De Melo \& Santos et al., 2014; Denisi \& Smith, 2014).

$\mathrm{Na}$ Tabela 2 são apresentados testes complementares ao teste de diferença de médias para os dois grupos de indivíduos, utilizando apenas dados de antes da realização de treinamentos, com o intuito de mitigar problemas de auto-seleção e verificar que os desempenhos desses indivíduos antes dos treinamentos eram iguais. Observase que como o coeficiente da variável treinamento não foi significativo, os desempenhos dos indivíduos que treinaram e não treinaram não eram diferentes no período anterior ao treinamento. Assim, indivíduos treinados e não treinados receberam a mesma média de avaliações antes dos treinamentos, o que mitiga e minimiza problemas associados à auto-seleção.

Tabela 2. Comparação do grupo de tratamento e não-tratamento antes do treinamento

Esta tabela apresenta resultados acerca das diferenças de desempenho de servidores que receberam o treinamento (grupo de tratamento) e não receberam o treinamento (grupo de não-tratamento), mas considerando-se apenas dados do período anterior à realização do treinamento. Especificamente, tais análises permitem minimizar as preocupações acerca do viés de seleção entre os grupos de servidores que se inscreveram e não se inscreveram para o treinamento. Para tanto, utilizou-se o modelo a seguir, o qual permitiu que fosse controlado ainda por outras características que possam ter afetado o desempenho, tais como autoavaliação do servidor, avaliação do usuário do serviço, e idade, tempo de serviço e gênero do servidor.

$$
\text { PARES }_{i t}=+\alpha_{\mathrm{i}}+\mu_{\mathrm{t}}+\text { Treinamento }_{\mathrm{i}}+\text { Controles }_{\mathrm{it}}+\varepsilon_{i t}
$$

\begin{tabular}{|c|c|c|c|c|}
\hline Variável & Coeficiente & Erro padrão & $\mathbf{T}$ & $P>t$ \\
\hline Treinamento & -1.158 & 1.068 & -1.08 & 0.278 \\
\hline Auto-Avaliação & 0.522 & 0.011 & 43.56 & 0.000 \\
\hline Avaliação-Usuário & 0.035 & 0.010 & 3.28 & 0.001 \\
\hline Idade & 0.025 & 0.027 & 0.92 & 0.359 \\
\hline Tempo Serviço & -0.029 & 0.032 & -0.92 & 0.358 \\
\hline Gênero & 1.881 & 0.378 & 4.98 & 0.000 \\
\hline
\end{tabular}

Número de observações $=2845$

Definição: PARES $_{\text {it }}=$ resultado da nota atribuída ao desempenho do indivíduo i no instante t por outros servidores do mesmo setor; Treinamento $=1$ para indivíduos que receberam o treinamento, 0 caso contrário; Auto-Avaliação $=$ percepção do próprio servidor sobre seu desempenho; Avaliação-Usuário =percepção média sobre o desempenho por parte dos usuários internos do serviço; Idade = idade do servidor; Tempo de Serviço = tempo de serviço do servidor na organização; Gênero $=1$ se Masculino e 0 se Feminino.

Fonte: Elaborada pelos autores. 


\subsection{Analisando o grupo de tratamento - antes e depois do treinamento}

Na Tabela 3, são mostrados os resultados de comparações entre indivíduos do grupo que participoude treinamento (Tratamento), considerando-se observações de todo o período analisado, antes e depois dos treinamentos. Observa-se que, em princípio, houve uma melhora no desempenho dos indivíduos após participarem de treinamentos. Contudo, características endógenas podem ter contribuído para essa melhoria, pois não foram analisados nessa regressãio dados do grupo de controle que pudessem eliminar tais características.

Efeitos de características endógenas podem ser verificados em diversos estudos, por exemplo, em De Grip, Sauermann (2012), que mostraram aumento de 10\% no desempenho de trabalhadores em atividade de Call Center após eles terem participado de treinamentos quando comparado o grupo de tratamento e o grupo de controle.

Tabela 3. Comparando antes e depois apenas para quem fez o treinamento

Testes aplicados antes e depois dos treinamentos, somente para indivíduos que participaram de treinamento, a partir do modelo exposto a seguir. Controlou-se neste modelo por outras características que possam ter afetado o desempenho, tais como: Auto-Avaliação; Avaliação- Usuário; Idade do servidor; Tempo de Serviço e Gênero. PARES $_{i t}=+\alpha_{i}+\mu_{t}+$ Tempo $_{t}$

+ Controles $_{\mathrm{it}}+\varepsilon_{i t}$

\begin{tabular}{lcccc}
\hline \multicolumn{1}{c}{ Variável } & Coeficiente & Erro padrão & T & P>t \\
\hline Tempo & 2.039 & 1.036 & 1.97 & 0.049 \\
Auto-Avaliação & 0.402 & 0.021 & 18.72 & 0.000 \\
Avaliação-Usuário & 0.025 & 0.014 & 1.73 & 0.084 \\
Idade & 0.020 & 0.037 & 0.54 & 0.588 \\
Tempo de Serviço & -0.034 & 0.075 & -0.46 & 0.649 \\
Gênero & 2.566 & 0.567 & 4.53 & 0.000 \\
Número de observações = 1134 & & & &
\end{tabular}

Definição: PARES $_{\text {it }}=$ resultado da nota atribuída ao desempenho do indivíduo i no instante t por outros servidores do mesmo setor; Tempo = 1 para depois do treinamento, 0 caso contrário; Auto-Avaliação = percepção do próprio servidor sobre seu desempenho; Avaliação-Usuário =percepção média sobre o desempenho por parte dos usuários internos do serviço;Idade = idade do servidor; Tempo de Serviço = tempo de serviço do servidor na organização; Gênero $=1$ se Masculino e 0 se Feminino.

Fonte: Elaborado pelos autoresr

Em outro experimento feito para estimar quanto o treinamento contribuiu para melhoria da produtividade individual, comparando dados de duas montadoras japonesas, Ariga et al. (2013) concluíram que resultados sobre o efeito de treinamento no trabalho variaram em razão da variável dependente utilizada, possibilitando a interpretação que características individuais, do local de trabalho e da relação entre os pares e supervisores podem alterar os resultados.

Além desses trabalhos, Magalhães et al. (2010), que tinham como objetivo identificar o modo como o processo de aperfeiçoamento de servidores técnico-administrativos influencia a realização das atividades em uma Instituição Pública de Ensino, constatou-se que diversos elementos interferem na dinâmica do processo, entre os quais a ausência de uma política de capacitação explícita, o que impactou negativamente os resultados esperados.

Logo, características endógenas podem afetar o desempenho e contribuir para o aumento do desempenho do grupo de servidores analisados, tal como na Tabela 3. Assim, não foi possível avaliar por meio da referida regressão o efeito direto do treinamento, uma vez que não se controlou pelo grupo de indivíduos não treinados. As próximas análises levam em consideração tais grupos.

\subsection{Efeitos do treinamento}

Na Tabela 4 são apresentados os resultados da estimação do modelo 1 considerando-se as duas especificações propostas. Primeiramente o desempenho de servidores que receberam o treinamento (grupo de tratamento) foi comparado ao desempenho ds servidores que não receberam o treinamento (grupo de não-tratamento). Em um 
segundo momento, o mesmo modelo foi estimado, mas considerando como grupo de controle apenas os servidores não treinados e que entraram no mesmo período que os servidores que receberam o treinamento (servidores que ingressaram depois de 2008).

Os resultados da Tabela 4 apresentam evidências de que o treinamento não teve efeito significativo sobre o desempenho dos servidores em ambas as especificações, dada a não significância estatística do coeficiente da interação entre as variáveis Treinamento e Tempo. Nesta Tabela são apresentados os resultados da estimação do modelo apresentado a seguir, considerando-se as duas especificações propostas. Primeiramente, o desempenho dos servidores que receberam o treinamento (grupo de tratamento) foi comparado ao desempenho dos servidores que não receberam o treinamento (grupo de não-tratamento). Em um segundo momento, o mesmo modelo foi estimado, mas considerando como grupo de controle apenas os servidores não treinados e que entraram no mesmo período que os servidores que receberam o treinamento (servidores que ingressaram depois de 2008). Nesse caso, os resultados obtidos suportam a Hipótese H1 de que treinamentos não necessariamente têm efeito positivo sobre o desempenho individual de servidores públicos.

Em particular, é possível que a melhoria do desempenho de servidores nas análises que não levaram em consideração o grupo de controle esteja associada ao ganho de experiência no tempo e não aos treinamentos (Aguinis \& Kraiger, 2009; Ariga et al., 2013; Fonseca et al., 2013). Tais resultados reforçam um dos principais problemas do serviço público brasileiro, cuja legislação trabalhista e características das Organizações são barreiras para a remuneração variável, deixando as organizações sob a influência do risco moral, uma vez que o desempenho dos trabalhadores fica condicionado às motivações pessoais em detrimento dos objetivos das organizações (Klann et al., 2015). Além disso, tal resultado levanta dúvidas acerca da qualidade ou necessidade da oferta de alguns treinamentos em instituições públicos, como é o caso do treinamento de ambientação (Cavazotte, De Assis Moreno Jr, \& Turano, 2015). Tais treinamentos consomem uma grande quantidade de recursos públicos e os resultados obtidos não evidenciam melhora no desempenho dos servidores.

Outro fator que corrobora com a ineficácia do treinamento com relação ao desempenho foi a criação da Ambientação como capacitação obrigatória, o que pode diminuir a motivação que leva o indivíduo a participar do treinamento e provocar atitudes menos favoráveis ao aprendizado (Salas et al., 2012). Nesse caso, aliado ao problema de risco moral causado pela ausência da política de incentivos, é possível que a ineficácia do treinamento possa estar associada à qualidade do ambiente organizacional. Em outras palavras, ainda que existissem mecanismos de incentivos ao aumento da produtividade, problemas no ambiente da instituição ou entre os servidores de um mesmo setor poderiam diminuir a eficácia percebida dos treinamentos.

Nesse contexto, a literatura aponta pela necessidade do desenvolvimento de políticas de planejamento e gestão do ambiente organizacional para a eficácia dos treinamentos. Isso porque o sucesso do treinamento não depende apenas dos mecanismos de incentivo, mas da qualidade do seu programa e das variáveis de contexto. A correlação entre as variáveis de qualidade que dão suporte à aprendizagem e à performance no trabalho, tal como analisado por Balarini, Zerbini e Martins (2014), corroboram a necessidade de conciliação de motivação, qualidade do treinamento e incentivos individuais para o sucesso de treinamentos.

Tabela 4. Efeito do treinamento sobre a percepção de desempenho

PARES $_{i t}=+\alpha_{\mathrm{i}}+\mu_{\mathrm{t}}+$ Treinamento $_{\mathrm{i}}+$ Treinamento $_{\mathrm{i}}$ Tempo $_{t}+$ Controles $_{\mathrm{it}}+\varepsilon_{i t}$

\begin{tabular}{lcccc}
\hline \multirow{2}{*}{ Variável } & $\begin{array}{c}\text { Tratamento x Não-Tratamento } \\
\text { Coeficiente }\end{array}$ & $\mathbf{P > t}$ & Tratamento x Controle \\
\hline Treinamento & -0.952 & 0.510 & 2.675 & $\mathbf{P}>\mathbf{t}$ \\
Treinamento.tempo & 1.083 & 0.455 & -2.846 & 0.189 \\
Auto-Avaliação & 0.489 & 0.000 & 0.458 & 0.145 \\
Avaliação-Usuário & 0.054 & 0.000 & 0.100 & 0.000 \\
Idade & 0.018 & 0.483 & 0.091 & 0.000 \\
Tempo Serviço & -0.013 & 0.693 & -0.108 & 0.014 \\
Gênero & 1.971 & 0.000 & 1.346 & 0.011 \\
Número de observações: & 3830 & & 1931 & \\
\hline
\end{tabular}


Definição: PARESit =resultado da nota atribuída ao desempenho do indivíduo i no instante t por outros servidores do mesmo setor; Treinamento $=1$ para indivíduos que receberam o treinamento, 0 caso contrário; Tempo = 1 para depois do treinamento, 0 caso contrário; Auto-Avaliação = percepção do próprio servidor sobre seu desempenho; Avaliação-Usuário = percepção média sobre o desempenho por parte dos usuários internos do serviço; Idade = idade do servidor; Tempo de Serviço = tempo de serviço do servidor na organização; Gênero = 1 se Masculino e 0 se Feminino.

Fonte: Elaborada pelo autor

Os resultados obtidos seguem alinhados às diretrizes que mencionam o treinamento não como um evento isolado e único, mas como um processo sistêmico, que, se bem projetado, funcionará como estímulo às várias métricas de bem-estar corporativo (Salas et al., 2012). Por isso, a formulação, a implementação e a avaliação de treinamentos devem ser conduzidas a partir de decisões adotadas visando à eficácia de treinamentos, tanto sob o ponto de vista dos mecanismos de incentivo quanto sob o ponto de vista da qualidade do treinamento e do ambiente organizacional.

Assim, reforça-se a constatação de que a eficiência da avaliação do desempenho produtivo do indivíduo será identificada mediante um programa completo de gestão do desempenho, que inclua todas as práticas e políticas organizacionais, além deobjetivos claros e métricas que não apresentem resultados distorcidos (Aguinis \& Pierce, 2008; Grumeman \& Saks, 2011; Speklé \& Verbeeten, 2014).

\section{CONSIDERAÇÕES FINAIS}

Em geral, os resultados obtidos nesta pesquisa sugerem que o treinamento analisado não teve impacto sobre o desempenho individual dos servidores treinados. Embora não possam ser estendidos a qualquer tipo de treinamento no setor público, tais resultados reforçam um dos principais problemas do serviço público brasileiro, cuja legislação trabalhista e características das organizações são barreiras para a remuneração variável e deixam as organizações sob a influência do risco moral, uma vez que o desempenho dos trabalhadores fica condicionado às motivações pessoais em detrimento dos objetivos das instituições.

Aliado ao problema de risco moral causado pela ausência da política de incentivos, é possível que a ineficácia do treinamento possa estar associada à qualidade do ambiente organizacional. Em outras palavras, ainda que existissem mecanismos de incentivos ao aumento da produtividade tais como a remuneração variável por desempenho, problemas no ambiente da instituição ou entre os servidores de um mesmo setor poderiam diminuir a eficácia percebida dos treinamentos.

Assim, considerando que a nova gestão pública está baseada em resultados, os resultados apontam para a necessidade de avaliação das metas e de resultados na agenda de trabalho das instituições públicas brasileiras e implementação programas de gestão de desempenho, baseados em objetivos claros e em métricas que não gerem resultados distorcidos, que englobem a complexidade de informações relativas às características individuais e organizacionais (Matei \& Antonie, 2014; Speklé \& Verbeeten, 2014).

Os resultados desta pesquisa não permitem fazer afirmações específicas sobre a qualidade dos treinamentos analisados. Entretanto, os números apresentados pelo CDS demonstram que o projeto representou, no período pesquisado, $70 \%$ dos treinamentos oferecidos pela instituição, com aproximadamente 2.430 horas de treinamentos. Neste contexto, embora não tenha gerado resultados significativos, o treinamento de ambientação representou um percentual significativo da política de treinamento de servidores da instituição. Em conjunto, tais resultados levantam dúvidas acerca da necessidade ou qualidade da oferta de alguns treinamentos em instituições públicas, como é o caso do treinamento de ambientação. Os resultados do treinamento investigado sugerem que tais treinamentos consomem uma grande quantidade de recursos públicos e os resultados obtidos não evidenciam necessariamente uma melhora no desempenho dos servidores.

Os resultados dessa pesquisa podem trazer contribuições para o IFES, colaborando com decisões sobre a implementação da política de capacitação de pessoal, que se encontra em fase de elaboração, por comissão designada pelo Reitor (IFES, 2016), bem como com um possível aprimoramento do programa de avaliação de desempenho, que poderá evoluir para o programa de gestão do desempenho, valorizando as competências acumuladas pelos servidores e também as obtidas em treinamentos.

Nesse contexto, é recomendável também que a área de Recursos Humanos, responsável pelas processos de 
capacitação e avaliação de desempenho de pessoal, alcance um papel estratégico, já que o sucesso de uma política integrada de recursos humanos é fundamental para o sucesso da implementação de programas de treinamento e de gestão do desempenho.

\section{REFERÊNCIAS}

Agrawal, C. (2013). Performance-related Pay-Hype versus Reality: With Special Reference to Public Sector Organizations. Management and Labour Studies, 37(4), 337-344.

Aguinis, H., \& Pierce, C. A. (2008). Enhancing the relevance of organizational behavior by embracing performance management research. Journal of Organizational Behavior, 29(1), 139-145.

Aguinis, H., \& Kraiger, K. (2009). Benefits of training and development for individuals and teams, organizations, and society. Annual review of psychology, 60, 451-474.

Amorim, T. N. G. F., \& de Barros Silva, L. (2013). Treinamento no Serviço Público: Uma Abordagem com os Servidores Técnico-Administrativos de Universidade. TPA-Teoria e Prática em Administração, 2(1), 1-28.

Ariga, K., Kurosawa, M., Ohtake, F., Sasaki, M., \& Yamane, S. (2013). Organization adjustments, job training and productivity: Evidence from Japanese automobile makers. Journal of the Japanese and International Economies, 27, 1-34.

Balsan, L. A. G., Lopes, L. F. D., Alves, J. N., Vizzotto, F. B., \& Costa, V. M. F. (2016). Impacto do treinamento, comprometimento e entrincheiramento organizacionais em servidores de uma universidade pública. Revista Gestão Universitária na América Latina-GUAL, 9(1), 143-164.

Bégin, L., \& Véniard, A. (2013). L'évaluation à $360^{\circ}$ : le rôle du feedback par les pairs comme outil de développement des compétences comportementales des futurs managers. Management \& Avenir, (4), 32-51.

Brandão, H. P., Borges-Andrade, J. E., \& Guimarães, T. D. A. (2012). Organizational performance and its relation with management competencies, perceived organizational support and training. Revista de Administração (São Paulo), 47(4), 523-539.

Brasil. Decreto $\mathrm{n}^{\mathrm{o}}$ 5.707, de 23 de Fevereiro de 2006. Institui a Política e as Diretrizes para o Desenvolvimento de Pessoal da administração pública federal direta, autárquica e fundacional, e regulamenta dispositivos da Lei no 8.112, de 11 de dezembro de 1990. Recuperado em 01 outubro, 2015, de http://www.planalto.gov.br/CCIVIL_03/Ato2004-2006/2006/Decreto/D5707.htm.

Brasil. Decreto $\quad \mathrm{n}^{\mathrm{o}}$ 5.824, de 29 dejunho de 2006. Estabelece as Diretrizes para Elaboração do Plano de Desenvolvimento dos Integrantes da Carreira dos Cargos Técnico-administrativos em Educação, instituído pela Lei $n^{\circ}$ 11.091, de 12 de janeiro de 2005. Recuperado em 01 outubro, 2015, de http://www.planalto.gov.br/ccivil 03/ ato2004-2006/2006/decreto/ d5825.htm.

Bresser-Pereira, L. C. (2010). Democracia, estado social e reforma gerencial. Revista de Administração de Empresas, 50(1), 112-116.

Carvalho, A.I. de. (2009). Escolas de governo e gestão por competências: mesa-redonda de pesquisa. Brasília: ENAP,

Cavazotte, F. D. S. C. N., Moreno Jr, V. D. A., \& Turano, L. M. (2015). Continuous learning culture, attitudes and job performance: comparing companies in public and private sectors. Revista de Administração Pública, 49(6), 1555-1578.

Chiaburu, D. S., Van Dam, K., \& Hutchins, H. M. (2010). Social support in the workplace and training transfer: A 
longitudinal analysis. International Journal of Selection and Assessment, 18(2), 187-200.

Coda, R., \& Coda, D. A. (2014). Desempenho Estratégico do Departamento de Gestão de Recursos Humanos: uma Pesquisa Exploratória Acerca das Implicações dos Estilos Comportamentais de seus Profissionais. BBRBrazilian Business Review, 11(4), 116-140.

Colombo, E., \& Stanca, L. (2014). The impact of training on productivity: evidence from a panel of Italian firms. International Journal of Manpower, 35(8), 1140-1158.

De Grip, A., \& Sauermann, J. (2012). The effects of training on own and co-worker productivity: Evidence from a field experiment. The Economic Journal, 122(560), 376-399.

De Grip, A., \& Sauermann, J. (2013). The effect of training on productivity: The transfer of on-the-job training from the perspective of economics. Educational Research Review, 8, 28-36.

DeNisi, A., \& Smith, C. E. (2014). Performance appraisal, performance management, and firm-level performance: a review, a proposed model, and new directions for future research. Academy of Management Annals, 8(1), $127-179$

Filardi, F., Freitas, A. S. D., Irigaray, H. A., \& Ayres, A. B. (2016). (Im) Possibilities for applying the Excellence in Public Management Model (MEGP). Revista de Administração Pública, 50(1), 81-106.

Fonseca, D. R., Meneses, P. P. M., da Silva Filho, A. I., \& Campos, N. G. (2013). Autonomia para gestão estratégica de pessoas no setor público federal: perspectivas de análise e agenda de pesquisa. Revista de Administração Pública, 47(6), 1451-1476.

Grossman, R., \& Salas, E. (2011). The transfer of training: what really matters. International Journal of Training and Development, 15(2), 103-120.

Guerrazzi, M. (2016). The effect of training on Italian firms' productivity: microeconomic and macroeconomic perspectives. International Journal of Training and Development, 20(1), 38-57.

Järvalt, J., \& Randma-Liiv, T. (2010). Public sector HRM: the case of no central human resource strategy. Baltic Journal of Management, 5(2), 242-256.

Junior, O. D. S. G., Zouain, D. M., \& de Oliveira Almeida, G. (2014). Competências e habilidades relevantes para um chefe de unidade descentralizada de perícia da Polícia Federal. Revista de Administração Mackenzie, 15(4).

Klann, R. C., Gomes, E. D. C. O., Greuel, M. A., \& Bezerra, F. A. (2015). Influência do risco moral e da accountability nas tomadas de decisões. Contabilidade Vista \& Revista, 25(1), 99-118.

Klering, L.R., Porsse, M.C.S., \& Guadagnin, L.A. (2010). Novos caminhos da Administração Pública Brasileira. Análise, Porto Alegre, 21(1), 4-17.

Koga, N. M. (2014). Gestão de pessoas na administração pública japonesa. Revista do Serviço Público, 58(1), 97-106.

Kumpikaite, V. (2015). Human resource training evaluation. Engineering economics, 5(55), 29-36.

Lopes, S. A., \& Teixeira, P. (2013). Productivity, wages, and the returns to firm-provided training: fair shared capitalism?. International Journal of Manpower, 34(7), 776-793.

Magalhães, E. M. D., Oliveira, A. R. D., Cunha, N. R. D. S., Lima, A. A. T. D. F., \& Campos, D. C. D. S. (2010). Training policy for the technical-administrative staff at the Federal University of Viçosa (UFV) as perceived by trained employees and managers. Revista de Administração Pública, 44(1), 55-86.

Martin, H. J. (2010). Workplace climate and peer support as determinants of training transfer. Human Resource Development Quarterly, 21(1), 87-104. 
Matei, A., \& Antonie, C. (2014). The new public management within the complexity model. Procedia-Social and Behavioral Sciences, 109, 1125-1129.

McNamara, T. K., Parry, E., Lee, J., \& Pitt-Catsouphes, M. (2012). The effect of training on organizational performance: differences by age composition and cultural context. The International Journal of Human Resource Management, 23(6), 1226-1244.

Melo Santos, N., Bronzo, M., de Oliveira, M. P. V., \& de Resende, P. T. V. (2014). Cultura organizacional, estrutura organizacional e gestão de pessoas como bases para uma gestão orientada por processos e seus impactos no desempenho organizacional. BBR-Brazilian Business Review, 11(3), 106-129.

Méndez, F., \& Sepúlveda, F. (2016). A Comparative Study of Training in the Private and Public Sectors: Evidence from the United Kingdom and The United States. Contemporary Economic Policy, 34(1), 107-118.

Nakayama, W. K., \& Salotti, B. M. (2014). Fatores determinantes do nível de divulgação de informações sobre combinações de negócios com a entrada em vigor do pronunciamento técnico CPC 15. Revista Contabilidade \& Finanças, 25(66), 267-280.

OCDE. (2010). PISA 2009Results: What Students Know and Can Do: Student Performance in Reading, Mathematics and Science (Volume I).

Pantoja, M. J., Iglesias, M., Benevenuto, R., \& Paula, A. D. (2012). Política nacional de desenvolvimento de pessoal na administração pública federal: uma caracterização da capacitação por competências na administração pública federal.

Patel, L. (2010). ASTD State of the industry report 2010. Alexandria, VA: American Society for Training \& Development.

Peci, A., Pieranti, O. P., \& Rodrigues, S. (2008). Governança e New Public Management: convergências e contradições no contexto brasileiro. Organizações \& Sociedade, 15(46), 39-55.

Poister, T. H., Thomas, J. C., \& Berryman, A. F. (2013). Reaching Out to Stakeholders: The Georgia DOT 360-Degree Assessment Model. Public Performance \& Management Review, 37(2), 302-328.

Salas, E., Tannenbaum, S. I., Kraiger, K., \& Smith-Jentsch, K. A. (2012). The science of training and development in organizations: What matters in practice. Psychological science in the public interest, 13(2), 74-101.

Speklé, R. F., \& Verbeeten, F. H. (2014). The use of performance measurement systems in the public sector: Effects on performance. Management Accounting Research, 25(2), 131-146.

Valdes, N., Mendez, F., \& Sepulveda, F. (2015). Legalization and Human Capital Accumulation.

Verbeeten, F. H., \& Speklé, R. F. (2015). Management control, results-oriented culture and public sector performance: Empirical evidence on New Public management. Organization studies, 36(7), 953-978. 